\title{
Analysis of Selective Versus Non-Selective Culture Media for Group B Streptococcus Isolation in Pregnancy
}

\author{
Sharmila Vijayan ${ }^{1 *}$ and Thirunavukkarasu Arun Babu ${ }^{2}$ \\ ${ }^{1}$ Additional Professor, Dept. of Obstetrics and Gynecology, All India Institute of medical Sciences (AIIMS), Mangalagiri, India \\ ${ }^{2}$ Associate Professor, Dept. of Pediatrics, All India Institute of Medical Sciences (AIIMS), Mangalagiri, India
}

\begin{abstract}
DOI: $10.36348 /$ sijog.2020.v03i07.003
| Received: 29.05.2020 | Accepted: 09.06.2020 | Published: 21.07.2020
\end{abstract}

*Corresponding author: Sharmila Vijayan

Abstract

Background: Maternal genital tract colonization with GBS during pregnancy is an important risk factor for neonatal GBS infections. Methodology: This prospective study was done to determine Group B streptococcus (GBS) colonization in pregnancy using selective and non-selective culture media. The total sample size was 300 pregnant women. In 200 women, vaginal and rectal swabs were collected and inoculated separately in non- selective blood agar media and in another 100 women, vaginal and rectal swabs were collected and inoculated separately in selective Todd-Hewitt broth media for GBS isolation. Results: The detection rate for GBS was more when Todd Hewitt Broth was used for initial inoculation than direct inoculation in blood agar. The overall GBS prevalence rate among pregnant women was $2.3 \%$ in our study. Conclusion: Our finding supports the current recommendations that inoculation in selective medium is fundamental to maximize GBS detection rates and should be employed by laboratories involved in screening of pregnantwomen.

Keywords: Group B Streptococcus, Non-selective culture media, Selective culture media.

Copyright @ 2020: This is an open-access article distributed under the terms of the Creative Commons Attribution license which permits unrestricted use, distribution, and reproduction in any medium for non-commercial use (NonCommercial, or CC-BY-NC) provided the original author and source are credited.

\section{INTRODUCTION}

Group B streptococcus (GBS) is an important organism causing early-onset neonatal sepsis $[1,2]$. The newborn acquires the infection during labor or in-utero by transmission from colonized maternal genital tract. Maternal genital tract colonization with GBS is the main risk factor for neonatal disease [3]. Maternal colonization rates vary from 4 to $30 \%[4,5]$ and the differences in colonization rate depend on the population studied and the laboratory methods used for GBS detection.Microbiological techniques must be designed to maximize detection rates. The use of selective Todd Hewitt Broth media is reported to be a sensitive and adequate method for GBS detection [4]. Sampling of vaginal and rectal sites for GBS culture is recommended to increase the isolation rates in pregnant women. We analyzed the culture methods for the detection of GBS from vaginal and rectal sites in pregnant women using Todd-Hewitt selective medium and non-selective blood agar medium.

\section{METHODOLOGY}

This was a prospective descriptive study done over a period of 20 months in a tertiary care hospital in South India. The study was performed after obtaining institute Ethics committee clearance. The study was approved by the research and ethics committee of the institute. Pregnant women in their second and third trimesters, attending the antenatal outpatient department (OPD) of the institute, were enrolled in the study. Women with a history of intake of antibiotics during the past 2 weeks, preexisting medical disorders complicating pregnancy, and women in labor were excluded from the study. Pregnant women who participated in the study signed an informed written consent. Vaginal and rectal swabs were obtained from 200 women and were separately cultured in nonselective $5 \%$ blood agar plates for 48 hours for GBS detection. In another 100 women, vaginal and rectal swabs were inoculated into Todd-Hewitt selective broth medium, incubated for 24 hours, and then subcultured for 24 hours in $5 \%$ blood agar plates. GBS was identified based on the colony morphology, beta 
hemolysis, characteristic biochemical reactions (catalase negative, sodium hippurate hydrolysis positive, CAMP test positive, bacitracin resistance) and was confirmed by latex agglutination test. Data entry and analysis were done using statistical software SPSS for Windows Version 16.0 (SPSS Inc, Chicago, IL, USA). Detection rates were compared between the two groups of pregnant women for each type of culture medium and each sampling site. Percentages were used for calculation and a $\mathrm{P}$ value $<0.05$ was considered statistically significant.

\section{RESULTS}

The overall GBS prevalence rate among pregnant women in our study was $2.3 \%$ ie., 7 out of 300 women were positive for GBS colonization. The details regarding the site of isolation and culture medium used with respect to the individual GBS positive cases are shown in Table 1. Five cases were positive for GBS from both rectal and vaginal swabs. One case each was positive for GBS from only vaginal site and only rectal site.

Table-1: Analysis of GBS positive cases

\begin{tabular}{|l|l|l|l|l|}
\hline & \multicolumn{5}{|l|}{ THB } & \multicolumn{2}{|c|}{ Blood agar } \\
\hline & $\begin{array}{l}\text { Vaginal } \\
\text { swab(n=100) }\end{array}$ & $\begin{array}{l}\text { Rectal } \\
\text { swab(n=100) }\end{array}$ & $\begin{array}{l}\text { Vaginal } \\
\text { swab(n=200) }\end{array}$ & $\begin{array}{l}\text { Rectal } \\
\text { swab(n=200) }\end{array}$ \\
\hline CASE 1 & + & + & & \\
\hline CASE 2 & + & + & & \\
\hline CASE 3 & + & + & & \\
\hline CASE 4 & + & + & + & + \\
\hline CASE 5 & & & - & + \\
\hline CASE 6 & & & + & - \\
\hline CASE 7 & & & & \\
\hline
\end{tabular}

Selective THB cultures were positive in 8 out of the 200 swabs (4\%) and Non-selective blood agar cultures were positive in 4 out of 400 swabs (1\%). The isolation rate for GBS was more when
Todd Hewitt Broth was used for initial inoculation than direct inoculation in blood agar. Table 2 shows the comparison of selective versus nonselective culture media for GBS isolation

Table-2: Comparison of selective THB versus non-selective blood agar culture media for GBS isolation

\begin{tabular}{|l|l|l|c|c|}
\hline $\begin{array}{l}\text { Culture } \\
\text { media }\end{array}$ & $\begin{array}{l}\text { No. of women } \\
\text { tested(n=300) }\end{array}$ & $\begin{array}{l}\text { No. of swabs taken } \\
\text { (vaginal + rectal swabs) }\end{array}$ & GBS isolation & $\begin{array}{l}\text { GBS isolation } \\
\text { from swabs }(\%)\end{array}$ \\
\hline $\begin{array}{l}\text { Blood } \\
\text { agar }\end{array}$ & 200 & $\begin{array}{l}\text { 200 vaginal + 200 } \\
\text { rectal swabs }\end{array}$ & $\begin{array}{l}\mathbf{4 / 4 0 0} \\
\text { inoculation }\end{array}$ & $\mathbf{1 \%}$ \\
\hline THB & 100 & $\begin{array}{l}\text { 100 vaginal + 100 } \\
\text { rectal swabs }\end{array}$ & $\begin{array}{l}\mathbf{8} / 200 \\
\text { inoculation }\end{array}$ & $4 \%$ \\
\hline
\end{tabular}

Analysis of GBS isolation rates from vaginal swabs using blood agar and Todd Hewitt broth is shown in Table 3. Significantly more GBS positivity was obtained from vaginal swabs when THB was used for culture in comparison to blood agar

Table-3: Isolation Rates of GBS from Vaginal Swabs

\begin{tabular}{|l|l|l|}
\hline MEDIA & NO. OF VAGINAL SWABS TAKEN & GBS isolation (N=6) \\
\hline Blood agar & 200 & $2(1 \%)$ \\
\hline THB & 100 & $4(4 \%)$ \\
\hline
\end{tabular}

Analysis of GBS isolation rates from rectal swabs using blood agar and Todd Hewitt broth is shown in Table 4. Significantly more GBS positivity was obtained from rectal swabs when THB was used for culture in comparison to blood agar.

Table-4: Isolation Rates of GBS from Rectal Swabs

\begin{tabular}{|l|l|l|}
\hline MEDIA & NO. OF RECTAL SWABS TAKEN $(n=300)$ & GBS $\quad$ isolation $(N=6)$ \\
\hline Blood agar & 200 & $2(1 \%)$ \\
\hline THB & 100 & $4(4 \%)$ \\
\hline
\end{tabular}

\section{DISCUSSION}

Screening of Group B streptococcus colonization is recommended for all pregnant women at 35 - 37 weeks and in women at risk of preterm delivery, such as those with preterm labor and preterm premature rupture of membranes [4]. Antibiotic prophylaxis of GBS colonized women, during labor, greatly reduces the risk of neonatal disease [5]. 
Todd-Hewitt selective medium enriched with gentamicin and nalidixic acid inhibits growth of Gramnegative bacteria, and it has greater sensitivity when compared to other nonselective media, such as blood or Granada agar [6]. The GBS detection rate was $4 \%$ of all the samples incubated in Todd-Hewitt medium and $1 \%$ of all the samples incubated in non selective blood agar medium. This finding supports the current view that selective culture medium is fundamental to maximize detection rates and should be employed by laboratories involved in screening of pregnant women [4].

A study that evaluated maternal colonization rates from developing countries found a GBSprevalence rate of $12.7 \%$. However, when considering only those studies in which adequate laboratory methods were used (use of selective culture medium and collection of vaginal specimens), this rate increases to $17.8 \%$. Inappropriate microbiological methods were used in almost half of the patients included in this analysis [7].

In the clinical scenario where GBS screening was done, a critical issue is the time needed to obtain reliable results from cultures, which is 48 hours when using the methods recommended by CDC. Preterm labor and premature rupture of membranes are high-risk situations for early-onset sepsis, for which adequate intrapartum antibiotic prophylaxis is necessary. Rapid identification tests would be more suitable as reliable and immediate results would be available, thereby avoiding unnecessary antibiotics prescription. The only FDA approved rapid test is a real-time PCR assay, which has high sensitivity and specificity [8].

The economic costs of universal maternal screening must be considered and the cost-benefit ratio favors GBS culture screening. More research is required in evaluation of this issue in developing countries, where the prevalence is much lower when compared to developed countries. Our study supports the current recommendations that inoculation in selective culture medium is fundamental to maximize GBS detection rates and should be employed by laboratories involved in GBS screening of pregnant women.

\section{CONCLUSION}

The use of Todd-Hewitt selective medium yielded greater detection rates than non-selective medium in our study. Practically, a single combined vaginal anorectal sample incubated in selective medium would be appropriate for GBS screening during pregnancy. This strategy would minimize laboratory costs without compromising maximum detection capacity, which is crucial for the prevention of earlyonset neonatal disease.

\section{REFERENCES}

1. Schuchat, A., Zywicki, S. S., Dinsmoor, M. J., Mercer, B., Romaguera, J., O'Sullivan, M. J., ... \& Levine, O. S. (2000). Risk factors and opportunities for prevention of early-onset neonatal sepsis: a multicenter case-control study. Pediatrics, 105(1), 21-26.

2. Hyde, T. B., Hilger, T. M., Reingold, A., Farley, M. M., O’Brien, K. L., \& Schuchat, A. (2002). Trends in incidence and antimicrobial resistance of early-onset sepsis: population-based surveillance in San Francisco and Atlanta. Pediatrics, 110(4), 690695.

3. Benitz, W. E., Gould, J. B., \& Druzin, M. L. (1999). Risk factors for early-onset group B streptococcal sepsis: estimation of odds ratios by critical literature review. Pediatrics, 103(6), e77e77.

4. Fultz-Butts, K., Gorwitz, R. J., Schuchat, A., \& Schrag, S. (2002). Prevention of perinatal group B streptococcal disease; revised guidelines from CDC.

5. Schuchat, A. (2001). Group B streptococcal disease: from trials and tribulations to triumph and trepidation. Clinical infectious diseases,33(6), 751-756.

6. Gupta, C., \& Briski, L. E. (2004). Comparison of two culture media and three sampling techniques for sensitive and rapid screening of vaginal colonization by group B streptococcus in pregnant women. Journal of clinical microbiology, 42(9), 3975-3977.

7. Stoll, B. J., \& Schuchat, A. (1998). Maternal carriage of group B streptococci in developing countries. The Pediatric infectious disease journal, 17(6), 499-503.

8. Picard, F. J., \& Bergeron, M. G. (2004). Laboratory detection of group B Streptococcus for prevention of perinatal disease. European Journal of Clinical Microbiology and Infectious Diseases, 23(9), 66567. 